\title{
411 - Implementing the STrAtegies for RelaTives (START) intervention in Portugal - preliminary findings
}

Fatima Urzal; Ana Quintão; Catarina Santos; Nuno Moura; Ana Banazol; Rui Martins, MD; Penny

Rapaport; Sarah Amador; Gill Livingston, MBChB, MD, FRCPsych; Manuel Gonçalves-Pereira

\section{Introduction}

As in other countries, Portuguese family caregivers have unmet needs regarding information and distress. START (STrAtegies for RelaTives) is a manual-based coping intervention for families of people with dementia, including coping strategies and stress-management components, by Livingston and colleagues (https://www.ucl.ac.uk/psychiatry/research/mental-health-older-people/projects/start). In the UK, START has been clinically effective, immediately and continuing even after 6-years, without increasing costs. Clinical training and supervision ensures treatment fidelity. In Portugal, these kind of interventions are less available and, when provided, are mostly supportive and fail to address coping strategies. Paradoxically, recruitment may also prove challenging.

\section{Objectives}

We describe the development of the Portuguese translation of START, incorporating guidance from the UK team, and a pilot study of delivery to family caregivers of people with dementia. We will also discuss the challenges of recruiting participants and delivering the intervention.

\section{Method}

We translated the START intervention and recruited family caregivers from neurology and psychiatry outpatients, in a central hospital in Lisbon. Our baseline assessment included the Hospital Anxiety and Depression Scale and the Zarit Burden Interview. The pilot is still ongoing at time of submitting, so we focus on recruitment, baseline assessments and process issues.

\section{Results}

During a three-month period, we recruited six caregivers. Five were primary caregivers (spouses or adult children) who had been caring for their relatives for 2 up to 10 years. Two caregivers met the international cutoff for clinically relevant affective disorder. The most frequent motivators for taking part were learning to communicate with their relatives and increasing knowledge to build community resources. Overall, the subjective impression of the therapist in charge is that the intervention seems acceptable and promising.

\section{Discussion/Conclusions}

This pilot study will eventually lead to an improved version of the Portuguese version of the START manual. So far, the intervention seems appropriate for selected caregivers in Portugal. However, response to striking unmet needs, particularly basic home support, may need to precede interventions like START. We look forward to concluding the intervention study and analyzing the implementation challenges, as a basis to inform a wider-scale trial. 